\title{
Electrophysiologic efficacy of irrigated bipolar radiofrequency in the clinical setting
}

\author{
Stefano Benussi, MD, PhD, ${ }^{\mathrm{a}}$ Andrea Galanti, MD, ${ }^{\mathrm{a}}$ Valerio Zerbi, ${ }^{\mathrm{b}}$ Ylenia A. Privitera, ${ }^{\mathrm{b}}$ \\ Ida Iafelice, $\mathrm{MD},{ }^{\mathrm{b}}$ and Ottavio Alfieri, $\mathrm{MD}^{\mathrm{a}}$
}

\begin{abstract}
Objective: Clinical success of atrial fibrillation ablation depends on persistent transmurality of the lesions. Although bipolar radiofrequency grants acute pulmonary vein isolation, the fate of such ablations in the clinical setting is unknown. We assessed postoperative pulmonary vein isolation up to 3 weeks after open chest bipolar radiofrequency ablation.
\end{abstract}

\begin{abstract}
Methods: Thirteen consecutive patients with mitral valve disease (mean age, $60 \pm 10$ years) and atrial fibrillation undergoing concomitant ablation with the BP2 bipolar device (Medtronic, Inc, Minneapolis, Minn) were enrolled for electrophysiologic assessment. During surgery, pairs of additional temporary wires were positioned on the right pulmonary veins and on the roof of the left atrium before bipolar ablation. Entrance block (abatement or disconnection of electrogram potentials) and exit block (no entrainment during pulmonary vein pacing) of the right pulmonary veins and of the free left atrium were assessed before and after ablation. After right pulmonary vein isolation was obtained, one additional encircling line was added. Electrophysiologic assessment was repeated before discharge and at 3 weeks.
\end{abstract}

Results: Baseline right pulmonary vein pacing threshold was $2.9 \pm 1.6 \mathrm{~mA}$. After $3 \pm 1$ encircling ablations, bidirectional block was attained in all pulmonary veins. At pre-discharge electrophysiologic study, complete isolation persisted in all cases. At 3 weeks, conduction block persisted in $11(85 \%)$ of 13 patients. All patients were discharged in sinus rhythm. At follow-up (19 \pm 7 months), 12 (92\%) of 13 patients were still free from atrial fibrillation.

Conclusions: Irrigated bipolar radiofrequency ablation provides acute transmurality after multiple ablations. However, total recovery of conduction occurred in $15 \%$ of the patients after 3 weeks. Repeated multiple ablations, possibly complemented by block validation, are suggested to help achieve durable transmurality with such technology. (J Thorac Cardiovasc Surg 2010;139:1131-6)

Durable transmurality of the ablation lines is key to the clinical success of atrial fibrillation ablation..$^{1-3}$ Despite the significant advances that characterized ablation technology in the past decade, linear uninterrupted lesions proved difficult to obtain. ${ }^{4}$ In particular, although all ablation devices may quite effectively yield transmural atrial scars when applied endocardially in the open heart, only bipolar radiofrequency appears to be reliably transmural when used epicardially on the beating heart. ${ }^{5}$ Nevertheless, although acute isolation of the pulmonary veins (PVs) after bipolar radiofrequency ablation has been shown both experimentally and in the clin-

\footnotetext{
From the Division of Cardiac Surgery, ${ }^{\mathrm{a}}$ Rehabilitation Unit, ${ }^{\mathrm{b}} \mathrm{S}$ Raffaele University Hospital, Milan, Italy.

Disclosures: Stefano Benussi reports consulting fees from Estech and lecture fees from St. Jude, Atricure, Medtronic, Cryocath, and Edwards. Valerio Cerbi and Ylenia Privitera were supported by a grant from Medtronic.

Read at the Thirty-fifth Annual Meeting of The Western Thoracic Surgical Association, Banff, Alberta, Canada, June 24-27, 2009

Received for publication June 19, 2009; revisions received Nov 7, 2009; accepted for publication Dec 28, 2009.

Address for reprints: Stefano Benussi, MD, PhD, Division of Cardiac Surgery, S Raffaele University Hospital, via Olgettina 60, 20132 Milan, Italy (E-mail: stefano. benussi@hsr.it).

0022-5223/\$36.00

Copyright (C) 2010 by The American Association for Thoracic Surgery

doi:10.1016/j.jtcvs.2009.12.039
}

ical setting, ${ }^{6-8}$ the late outcome of such ablations has never been investigated in patients.

We evaluated the electrophysiologic properties of lesions performed with an irrigated bipolar radiofrequency device up to 3 weeks after surgery to document the fate of the resulting ablations after the acute phase.

\section{PATIENTS AND METHODS}

Concomitant atrial fibrillation treatment with bipolar radiofrequency was performed in 13 patients with mitral valve disease from October 2006 to May 2007. Indications for concomitant ablation were permanent atrial fibrillation in 6 patients and persistent atrial fibrillation in 7 patients. Preoperative data are summarized in Table 1.

The study protocol was approved by our institutional review board, and informed written consent was signed by each patient before the operation. All enrolled patients agreed to stay at our institution until completing the postoperative rehabilitation period and to keep the pacing wires until the end of such period (generally, a couple of additional weeks).

Preoperative coronary angiography was performed in all patients.

\section{Surgical Procedure}

All ablations were carried out using the BP2 irrigated bipolar radiofrequency device (Medtronic, Inc, Minneapolis, Minn), activated by a Cardioblate Generator (model 60890; Medtronic). PV isolation was accomplished on the beating heart after institution of normothermic cardiopulmonary bypass. After dissection of the pericardial reflections, the bipolar device 


\section{Abbreviations and Acronyms \\ $\mathrm{DC}=$ direct-current \\ $\mathrm{PV}=$ pulmonary vein \\ $\mathrm{RPV}=$ right pulmonary vein}

was clamped around the atrial cuff containing the inflow of the right pulmonary veins (RPVs) and radiofrequency energy was deployed (Figure 1). After each ablation, the clamp was released. Additional parallel ablations were performed as needed to achieve conduction block. One extra encircling ablation was then added. The heart was lifted, the Marshall ligament was interrupted, and a similar encircling ablation was performed on the left pulmonary cuff. After cardioplegic arrest, one endoepicardial line connecting the two encircling ablations was performed through the atriotomy, generally on the roof of the left atrium. Connecting lines were also performed to the appendage and to the mitral valve annulus with bipolar radiofrequency only. ${ }^{9}$ The ostium of the left atrial appendage was sutured from inside in all patients. Finally, the valve disease was corrected.

\section{Electrophysiologic and Clinical Assessment}

Pairs of additional temporary wires (model 6494F; Medtronic) were transfixed on the RPVs, close to their atrial ostia (Figure 1), and on the roof of the left atrium, in proximity to the Bachmann bundle, during surgery. We chose to test only the RPV because that was technically convenient.

After induction of general anesthesia, all patients underwent transesophageal echocardiography to rule out an intracavitary thrombus.

As part of the study protocol, after heparin administration, 10 of 13 patients who were in atrial fibrillation at this time were administered direct-current (DC) shock to allow for electrophysiologic assessment. Interestingly, virtually any intrapericardial spot of the RPVs that we could reach allowed us to get effective entrainment at low thresholds.

Pacing thresholds were assessed at baseline, after two parallel ablations around the RPV couple, and then after each additional ablation until total abatement of entrainment at $20 \mathrm{~mA}$, the maximum atrial output of the external temporary pulse generator that we used (model 5833; Medtronic). The same assessment was then repeated after chest closure and after removal of chest drains, to rule out possible dislodgment of the wires, and then before discharge and 3 weeks after the operation, before leaving the rehabilitation unit of our hospital.

Pacing thresholds from free left atrial electrodes were also tested at the same time points as control. In general, exit block was considered significant for pacing thresholds of $10 \mathrm{~mA}$ or greater. ${ }^{10}$ Entrance block, defined as the abatement or disconnection of electrogram potentials from the RPV leads, was also assessed acutely and at discharge using a portable Cardiolab-II polygraph (Prucka Engineering GE, Houston Tex). Entrance block recordings at 3 weeks were not available owing to the logistical problems related to the limited maneuverability of the recording apparatus.

After completion of the 3-week baseline study, the wires were removed. A transthoracic echocardiogram was then performed the next day, before discharge from the rehabilitation unit.

Twelve-lead electrocardiogram, 24-hour Holter monitoring, and transthoracic echocardiography were performed 3, 6, and 12 months after the operation and then on a yearly basis.

\section{Data Analysis}

Statistical analysis was carried out using the JMP 7.0 package (SAS Institute, Inc, Cary, NC). All data are generally expressed as mean \pm standard deviation (range) for continuous variables and as $\mathrm{N}(\%)$ for categorical variables. Nonnormally distributed variables, as assessed by the Kolmogorov-Smirnov test, are expressed as median and 25th to 75 th percentile
TABLE 1. Preoperative data

\begin{tabular}{lc}
\hline \multicolumn{1}{c}{ Variables } & $\mathbf{N}$ \\
\hline No. of patients & 13 \\
Age (y) & $60 \pm 10$ \\
Female gender & $7(54 \%)$ \\
Duration of atrial fibrillation (y)* & $1 ; 0.46-6$ \\
Atrial fibrillation type & \\
$\quad$ Permanent & $6(46 \%)$ \\
$\quad$ Persistent & $7(54 \%)$ \\
Valvular heart disease & $13(100 \%)$ \\
$\quad$ Mitral & $13(100 \%)$ \\
$\quad$ Tricuspid & $7(54 \%)$ \\
Left atrial dimension (mm) & $50 \pm 5.5$ \\
Telediastolic left ventricular diameter (mm) & $53.5 \pm 6.6$ \\
Ejection fraction (\%) & $60.7 \pm 4.6$ \\
\hline
\end{tabular}

Values are expressed as number and (percentage) for discrete variables and as mean \pm standard deviation for continuous variables. *Data are not normally distributed, so variables are expressed as: median; first quartile-third quartile.

unless otherwise stated. Possible variations of mean values of the pacing thresholds—expressed in milliamperes ( $\mathrm{mA}$ ) —over time were investigated with the analysis of variance. We then used the Tukey-Kramer single-step multiple comparison method to find which means were significantly different from one another.

\section{RESULTS}

The main surgical procedure was mitral valve replacement in 7 patients, repair in 6, and associated tricuspid annuloplasty in 7 patients. The mean cardiopulmonary bypass duration was $91 \pm 12$ minutes (range, 73-114 minutes), with a mean aortic crossclamp time of $70 \pm 11$ minutes (range, 54-93 minutes). All patients survived, without any complication. The mean hospital stay was $7 \pm 2$ days (range, $4-12$ days).

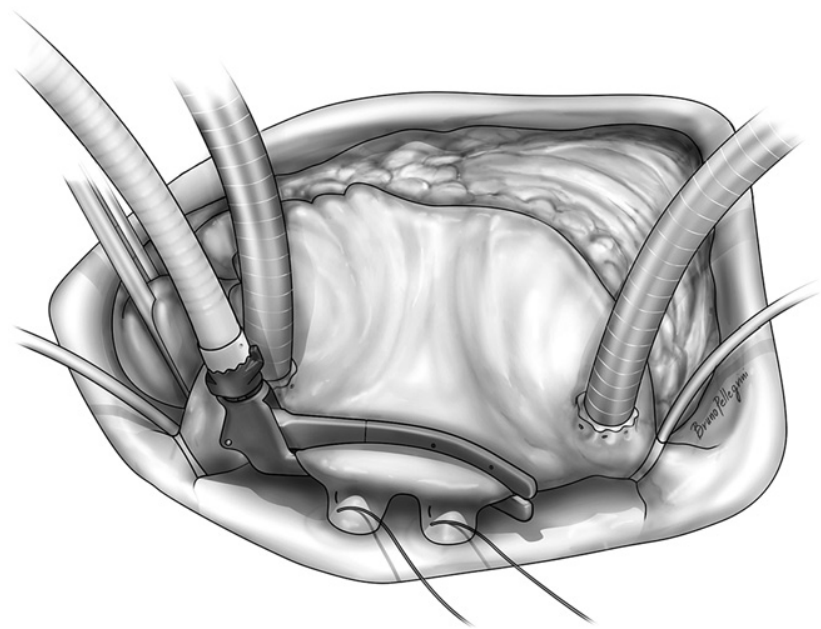

FIGURE 1. Right pulmonary vein ablation performed by irrigated bipolar radiofrequency device after positioning of a pair of temporary wires on the right pulmonary veins. 


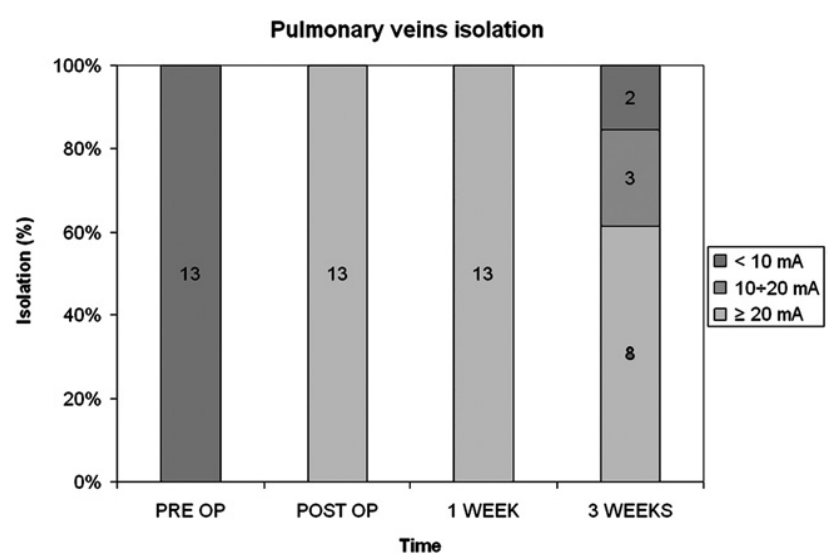

FIGURE 2. Pulmonary veins isolation status before and after bipolar radiofrequency ablation, before discharge, and at 3 weeks.

\section{Findings at Electrophysiologic Study}

During surgery, the baseline RPV pacing threshold was $2.9 \pm 1.6 \mathrm{~mA}$ (range, $0.8-6 \mathrm{~mA}$ ). RPV isolation, confirmed by bidirectional block, was obtained in all patients after $3 \pm$ 1 (range, 2-5) encircling ablations. As part of the study protocol, one additional ablation was then performed in all cases to consolidate conduction block.

Absence of entrainment at $20 \mathrm{~mA}$ persisted in all patients at chest closure and after removal of chest drains. At predischarge electrophysiologic study $(5.2 \pm 1.8$ days after surgery), complete isolation persisted in all cases, with no entrainment at $20 \mathrm{~mA}$. At 3 weeks' study ( $21 \pm 2.4$ days $)$, 2 patients showed no residual block across the RPVs encircling ablations (pacing thresholds 2.5 and $5 \mathrm{~mA}$, respectively). Of those $(11 / 13,85 \%)$ who showed a conduction block above $10 \mathrm{~mA}, 8$ had no entrainment at all $(20 \mathrm{~mA})$ and 3 had a pacing threshold of 10,14 , and $15 \mathrm{~mA}$, respectively (Figure 2).

The number of ablations required to achieve isolation was $2.5 \pm 0.7$ (respectively, 2 and 3 ) in the 2 patients with a totally recovered conduction and $3.1 \pm 1$ in the 11 remaining patients $(P=$ not significant $)$.

The mean pacing threshold from the RPV wires decreased significantly from $20 \mathrm{~mA}$ acutely and at 1 week to $15.9 \mathrm{~mA}$ at 3 weeks $(P<.05)$. Conversely, the pacing thresholds from the roof of the left atrium showed a slight but significant increase over time, from $0.9 \pm 0.5 \mathrm{~mA}$ acutely, to $1.2 \pm 0.5$ $\mathrm{mA}$ at 1 week, and to $1.9 \pm 1 \mathrm{~mA}$ at 3 weeks $(P<.05$, with significant increases at each following time point), compatible with a normal fibrous reaction around the electrodes.

The recording of RPV potential showed total disconnection of the electrical activity within the ablated cuff (Figure 3) or electrical silence in all the patients, up to 1 week after surgery. At completion of the last electrophysiologic assessment, all the electrodes were removed without any problem.

\section{Follow-up}

All patients were discharged in sinus rhythm.

At a mean follow-up of $19 \pm 7$ months (median, 21; 12 26 months) $12(92 \%)$ of 13 patients were in sinus rhythm. Of these, $6(50 \%)$ were free from antiarrhythmic medications, 4 receiving sotalol, 1 receiving amiodarone, and 1 receiving flecainide. Two patients required DC shock at 2 and at 9 months after surgery.

There was no clear-cut correlation between the RPV isolation status and clinical outcome. In particular, of the 2 patients with absent isolation, 1 received DC shock at 9 months and was in sinus rhythm with medications thereafter and the other did not have any recurrence.

\section{DISCUSSION}

Acute conduction block was always achieved after irrigated bipolar radiofrequency, but only after multiple parallel ablations. Furthermore, although a durable conduction block was maintained in most patients, we observed complete PV reconnection in $15 \%$ of the patients 3 weeks after surgery.

Owing to their unequalled efficacy, user friendliness, and safety profile, bipolar devices have become increasingly popular among surgeons in recent years. ${ }^{11}$ Nevertheless, clinical data on the electrophysiologic properties of ablations performed with bipolar radiofrequency are scarce. In particular, the post-acute fate of bipolar radiofrequency ablations in the clinical setting has never been reported. Therefore, our results provide important insights into the incidence, time course, and characteristics of recovery of PV conduction after bipolar ablation.

Prasad and associates ${ }^{12}$ showed dry bipolar radiofrequency to reproducibly yield transmural atrial ablations in dogs. In a similar acute study on ovine hearts, ablation with irrigated bipolar radiofrequency proved to yield a complete conduction block in $77 \%$ of the cases. ${ }^{4}$ Likewise, in the clinical setting, both impedance-guided and temperature-guided bipolar devices may require more than one ablation to achieve acute conduction block. ${ }^{6-8}$

Our results suggest that acute conduction block is not constantly achieved even after two parallel ablations with irrigated bipolar radiofrequency. Five parallel ablations were necessary to achieve acute block in 1 (of 13) patient with particularly thick tissue around the PV ostia.

Thirty days after ablation with dry impedance control radiofrequency, Prasad and colleagues ${ }^{12}$ found persistent isolation in 5 of 5 dogs. In another similar long-term study, the same group described irrigated bipolar radiofrequency to yield total isolation up to 1 month after ablation. ${ }^{13}$

In our study, despite proven isolation at surgery and at discharge, not all the PV couples showed a persistent conduction block at 3 weeks. A trend toward conduction recovery, which was complete in $2(15 \%)$ of 13 patients, seems to be confirmed by the fact that in another $23 \%$ of 

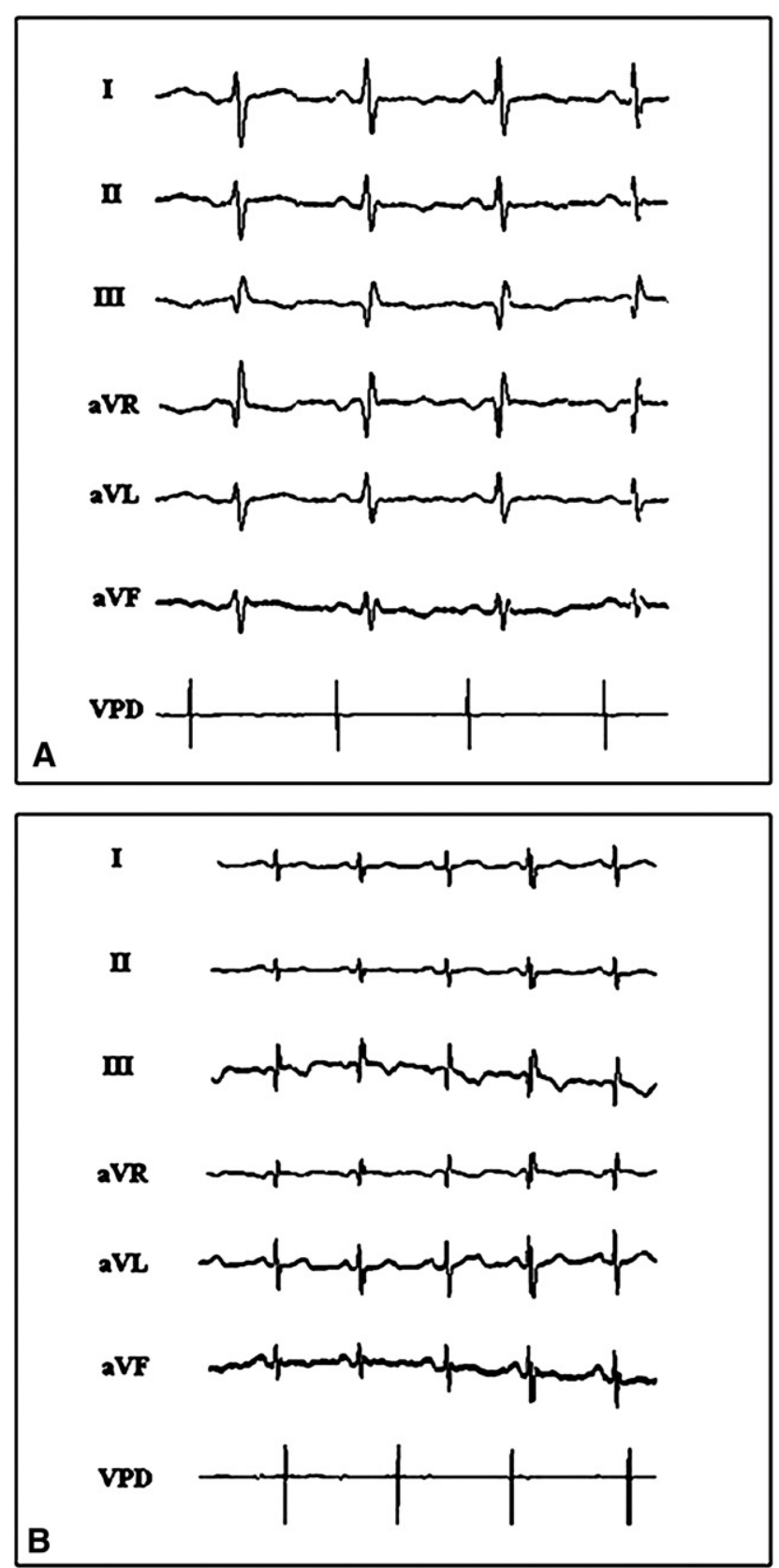

FIGURE 3. Right pulmonary vein electrogram showing activation synchronous with atrial depolarization (A) before ablation. Postoperative recording showing total disconnection between electrical activity in the pulmonary vein cuff and surface electrocardiogram (B). VPD, Ventricular premature depolarization.

the patients the pacing thresholds declined somewhat from $20 \mathrm{~mA}$ or more to 10 to $20 \mathrm{~mA}$ (Figure 2). In contrast, the pacing threshold from the control leads, positioned on the unablated roof of the left atrium, displayed a slight, but significant, increase, which is compatible with a normal "foreign body" fibrous reaction around the leads.

Late changes in lesion integrity may not be totally predictable on the basis of short-term testing. However, in the majority of cases the short-term result predicts long-term isolation of PV. ${ }^{1,2}$ The mechanism of PV reconnection is not clearly understood. Presumably, small gaps around the pulmonary antrum can be masked by edema owing to heart trauma. Resolution of swelling during recovery from ablation may allow PV reconnection.

Considerable evidence supports the relationship between electrical isolation and durable clinical success. ${ }^{14,15}$ The major predictor of arrhythmia recurrence after catheter ablation is absent $\mathrm{PV}$ isolation, indicating resumption of $\mathrm{PV}$ conduction or ineffective initial PV disconnection. ${ }^{2}$ Although an incomplete ablation around the $4 \mathrm{PVs}$ could lessen the inducibility of atrial fibrillation in a series of dogs, only a complete "box line" could totally abolish it. ${ }^{16}$ Such direct cause-effect relationship between PV inducibility and cure rate supports the central role of durable transmurality of the ablations in atrial fibrillation treatment.

Nevertheless, in our study, freedom from arrhythmia recurrence did not correlate with persistent PV isolation. This is possibly explainable considering the small sample size. Furthermore, of all the ablations composing a complete left lesion set, thus possibly influencing rhythm outcome, only the RPV encircling ablation was investigated in our study. It is noteworthy that while nontransmural epicardial ablation may prove partially beneficial owing to slowing of electrical conduction, to autonomic nervous system modulation, and other possible unknown mechanisms, it can also induce automatic iatrogenic arrhythmias that are usually hard to treat. ${ }^{17-19}$ This notwithstanding, most clinical reports on new ablation devices tend to focus on gross clinical outcomes rather than addressing simple electrophysiologic end points, such as acute conduction block.

Bipolar radiofrequency appears to be the most reliable way to get transmural ablations. Clamping allows an improved tissue-catheter contact, it clears out convective cooling exerted by circulating blood, and therefore results in ablations that are deeper and more continuous than those produced by unipolar ablation. ${ }^{4,5} \mathrm{PV}$ isolation with bipolar radiofrequency has been lately proposed as the last frontier of lone paroxysmal and recent-onset persistent atrial fibrillation. ${ }^{20}$ Techniques of biatrial extensive ablation using bipolar radiofrequency alone or in combination with cryoablation play a central role in the modern surgical treatment of long-standing persistent atrial fibrillation. ${ }^{8,21}$

Although impedance and temperature feedbacks featured by bipolar radiofrequency devices improve the reproducibility of complete tissue penetration, a completed ablation does not reliably translate into an isolating line of block. On the basis of our findings, blind single or double ablation of the PVs with irrigated bipolar radiofrequency may well turn into incomplete acute isolation. Moreover, acutely isolated segments may recover conduction once edema and reversible cell damage wear off. 
For practical purposes, it is then worth mentioning that complications related to overablation of the left atrium with bipolar radiofrequency-such as spontaneous wall rupture or pulmonary vein stenosis-have not been reported so far.

Therefore, either pacing validation of acute block, followed by $(>2)$ additional ablations or, alternatively, multiple (at least 5 or 6) parallel "blind" ablations, might be necessary to grant durable PV isolation after epicardial ablation with irrigated bipolar radiofrequency.

We are grateful to Professor Bruno Pellegrini for contributing the artwork. This work would not have been the same without the contribution of Professor M. A. Mariani, whom we owe for his knowing review and valuable suggestions.

\section{References}

1. Cheema A, Dong J, Dalal D, Marine JE, Henrikson CA, Spragg D, et al. Incidence and time course of early recovery of pulmonary vein conduction after catheter ablation of atrial fibrillation. J Cardiovasc Electrophysiol. 2007;18:387-91.

2. Verma A, Kilicaslan F, Pisano E, Marrouche NF, Fanelli R, Brachmann J, et al. Response of atrial fibrillation to pulmonary vein antrum isolation is directly related to resumption and delay of pulmonary vein conduction. Circulation. 2005; 112:627-35.

3. Todd DM, Skanes AC, Guiraudon G, Guiraudon C, Krahn AD, Yee R, et al. Role of the posterior left atrium and pulmonary veins in human lone atrial fibrillation: electrophysiological and pathological data from patients undergoing atrial fibrillation surgery. Circulation. 2003;108:3108-14.

4. Bugge E, Nicholson IA, Thomas SP. Comparison of bipolar and unipolar radiofrequency ablation in an in vivo experimental model. Eur J Cardiothorac Surg. 2005;28:76-80.

5. Aupperle H, Doll N, Walther T, Ullmann C, Schoon HA, Mohr WF. Histological findings induced by different energy sources in experimental atrial ablation in sheep. Interact Cardiovasc Thorac Surg. 2005;4:450-5.

6. Benussi S, Nascimbene S, Calori G, Denti P, Ziskind Z, Kassem S, et al. Surgical ablation of atrial fibrillation with a novel bipolar radiofrequency device. J Thorac Cardiovasc Surg. 2005;130:491-7.

7. Prasad SM, Maniar HS, Schuessler RB, Damiano RJ Jr. Chronic transmural atrial ablation by using bipolar radiofrequency energy on the beating heart. $J$ Thorac Cardiovasc Surg. 2002;124:708-13.

8. Gaynor SL, Diodato MD, Prasad SM, Ishii Y, Schuessler RB, Bailey MS, et al. A prospective, single-center clinical trial of a modified Cox maze procedure with bipolar radiofrequency ablation. J Thorac Cardiovasc Surg. 2004;128: 535-42.

9. Benussi S, Nascimbene S, Galanti A, Fumero A, Dorigo E, Zerbi V, et al. Complete left atrial ablation with bipolar radiofrequency. Eur J Cardiothorac Surg. 2008;33:590-5.

10. Gerstenfeld EP, Dixit S, Callans D, Rho R, Rajawat Y, Zado E, et al. Utility of exit block for identifying electrical isolation of the pulmonary veins. J Cardiovasc Electrophysiol. 2002;13:971-9.

11. Gammie JS, Sheng S, Griffith BP, Peterson ED, Rankin JS, O'Brien SM, et al. Trends in mitral valve surgery in the United States: results from the Society of Thoracic Surgeons Adult Cardiac Surgery Database. Ann Thorac Surg. 2009; 87:1431-7.

12. Prasad SM, Maniar HS, Diodato MD, Schuessler RB, Damiano RJ Jr. Physiological consequences of bipolar radiofrequency energy on the atria and pulmonary veins: a chronic animal study. Ann Thorac Surg. 2003;76:836-42.

13. Melby SJ, Gaynor SL, Lubahn JG, Lee AM, Rahgozar P, Caruthers SD, et al. Efficacy and safety of right and left atrial ablations on the beating heart with irrigated radiofrequency energy: a long-term animal study. $J$ Thorac Cardiovasc Surg. 2006;132:853-60.

14. Cappato R, Negroni S, Pecora D, Bentivegna S, Lupo PP, Carolei A, et al. Prospective assessment of late conduction recurrence across radiofrequency lesions producing electrical disconnection at the pulmonary vein ostium in patients with atrial fibrillation. Circulation. 2003;108:1599-604.

15. Callans DJ, Gerstenfeld EP, Dixit S, Zado E, Vanderhoff M, Ren JF, et al. Efficacy of repeat pulmonary vein isolation procedures in patients with recurrent atrial fibrillation. J Cardiovasc Electrophysiol. 2004;15:1050-5.
16. Van Brakel TJ, Bolotin G, Nifong LW, Dekker AL, Allessie MA, Chitwood WR Jr, et al. Robot-assisted epicardial ablation of the pulmonary veins: is a completed isolation necessary? Eur Hear J. 2005;26:1321-6.

17. Thomas SP, Johnson DC, Uther JB, Ross DL. Atrial flutter following a simplified maze procedure for cure of atrial fibrillation. Asia Pacific Heart J. 1998;7:103-7.

18. Usui A, Inden Y, Mizutani S, Takagi Y, Akita T, Ueda Y. Repetitive atrial flutter as a complication of the left-sided simple maze procedure. Ann Thorac Surg. 2002;73:1457-9.

19. Melby SJ, Lee AM, Zierer A, Kaiser SP, Livhits MJ, Boineau JP, et al. Atria fibrillation propagates through gaps in ablation lines: implications for ablative treatment of atrial fibrillation. Heart Rhythm. 2008;5:1296-301.

20. Wolf RK, Schneeberger EW, Osterday R, Miller D, Merrill W, Flege JB, et al. Video-assisted bilateral pulmonary vein isolation and left atrial appendage exclusion for atrial fibrillation. J Thorac Cardiovasc Surg. 2005;130:797-802.

21. Benussi S, Galanti A, Nascimbene S, Fumero A, Dorigo E, Zerbi V, et al. Right atrial ablation with bipolar radiofrequency. Ann Thorac Surg. 2009;87:1573-6.

\section{Discussion}

Kent W. Jones (Salt Lake City, Utah). I enjoyed this paper and I compliment Dr Benussi on his excellent study. I am aware of his interest in atrial fibrillation and the surgical treatment thereof. I think he spent a period of time with the pioneer in that field, Jim Cox, and I also think papers of this sort of very important in broadening our understanding of the surgical options for treating arrhythmias.

As you probably know, in the past year an article was published reviewing more than 62,000 patients from the Society of Thoracic Surgeons Database who went to the operating room for a cardiac surgical procedure with a known history of, or presently in, atrial fibrillation. Only $38 \%$ of those patients had a surgical ablative procedure, about $50 \%$ of the patients with mitral valve disease and only about $25 \%$ of patients with aortic valve disease or coronary disease. There is a huge patient population that we as cardiac surgeons are not addressing.

I agree that it is important, if one plans to measure exit block and entrance block, to place two electrodes on the RPV and two electrodes on the left atrium in an attempt to avoid one of the possible pitfalls that you outlined in your paper, that being the loss of conduction and therefore not really monitoring whether there is exit block or entrance block.

One question or disagreement I have with the terminology in your paper is that of using the word "transmurality," which is really a histologic term rather than an electrical term. You state that you found evidence of acute conduction block $100 \%$ of the time after crushing tissue with a clamp and heating that tissue. I think this is the description of a change in tissue impedance rather than a really adequate description or evidence of transmural injury.

I have a couple of questions, and I will preface them by a couple of statements or quotes from your paper.

One statement says that the clinical success of atrial fibrillation ablation depends on persistent transmurality of the lesions. I totally agree with that. Then you state that there is no correlation between the number of ablations required to obtain isolation and the final isolation result at 3 weeks. You also state that there was no clearcut correlation between RPV isolation status and clinical outcome evidenced by the fact that $85 \%$ of the patients had conduction block at 3 weeks, meaning that $15 \%$ of those patients had lost their conduction block. However, $92 \%$ of those patients were in normal sinus rhythm. 
My first question: Of the $15 \%$ of patients who had lost conduction block, what percent were in that group found to be in normal sinus rhythm?

Dr Benussi. Actually, of the two patients who reverted to preoperative pacing thresholds, one required DC shock and was in sinus rhythm thereafter. The other patient with total recovery of conduction fared perfectly after surgery, with stable sinus rhythm and no need of DC shock. That told us that in such a population of patients it is not possible to draw any meaningful inference on a possible correlation between electrophysiologic appropriateness of the ablation and sinus rhythm recovery.

First of all, there were only 13 patients. Second, because we only assessed RPV isolation, we did not know anything about the other 4 or 5 ablations that characterized the treatment of these patients.

Dr Jones. Second, the majority of the papers that you cited in the first part of your presentation were papers from the catheterization laboratory dealing with transcatheter ablation. I can understand the need for measuring exit block or block in conduction in those patients in whom one is using a multiple catheter technique. However, when one uses a device such as you described with a clamp across the PVs, and given the lack of correlation that you showed between the number of applications versus success, and with the evidence that you showed that there is certainly a disconnect between the patients with conduction block or loss thereof and the patients at 3 weeks in normal sinus rhythm, do you still think there is a need for measuring conduction block in the operating room knowing that the chance of getting $100 \%$ with the type of bipolar devices we use is very, very good. Why use the time?

Dr Benussi. I think we have two possible ways to proceed on that: First, we could validate the lines on a routine basis, which would make our procedures slightly more complex. However, once it becomes widely accepted that the electrophysiologic appropriateness of our ablation procedure translates itself into better rhythm outcomes, we could well spend those couple of additional minutes doing PV pacing. The second possibility is that we base our standard ablation practice on our preliminary results with intraoperative validation in a pilot initial series of patients. For instance, if you are using a device that after two parallel ablations proved to yield total block in $100 \%$ of your patients, you could pretty well rely on blind double ablation in your standard practice. I am afraid that by not paying attention to these very simple tricks, we will spoil the enormous advantage the cardiac surgeon has in terms of efficacy of the technology with respect to the electrophysiologists.

Dr Jones. Would it be of interest to do a randomized study measuring exit and/or entrance block in half of these patients, while measuring neither in the control group, and then comparing the results using the same time frames as cited in your presentation?
Dr Benussi. That is a good point. However, as said, in a study like ours, where only one little piece of the whole left ablation procedure is validated, it is difficult to draw general inferences on the importance of transmurality in determining the resulting rhythm outcome. But the evidence is already out there. Many studies on percutaneous AF ablation found incomplete isolation of the PVs to be the major predictor of arrhythmia relapse at follow-up. I think that, far from being enough, an appropriate PV isolation should be at least the minimal requirement of our procedures as well.

Dr Jones. I have two other very short questions. You said that you performed electrocardiography, Holter monitoring, and transthoracic echocardiography at 3 months, 6 months, and 12 months, but you gave no data as to the outcome. Are the patients the same? Are $92 \%$ of them still in normal rhythm at a year?

Dr Benussi. Correct. That was the result from the Holter monitoring, which is the base requirement for follow-up, according to the recommendations of the Heart Rhythm Society consensus.

Dr Jones. I have one short technique question. As I understand it, you use a purse-string suture on the inside of the left atrium to obliterate the appendage. Given the data from the Cleveland Clinic recently showing that $22 \%$ of those patients still had an hourglass narrowing at the site of appendage ligation, do you still use that procedure?

Dr Benussi. No, I have never used a purse-string suture on the appendage at all. I think the results from the Cleveland Clinic attest to two very important conclusions. First, you cannot do a purse-string suture and go away with that very easily. We do a double-layer mattress followed by over-and-over continuous suture of the appendage very meticulously, spending 2 to 3 minutes on that. Second, you should not expand too much the indications to prophylactic appendage closure because, actually, endoleaks are a potential problem and, of course, a partially occluded left appendage is the worse case scenario as far as the stroke risk is considered.

Dr Jason Bowles (St George, Utah). I have one quick question on the technical aspects. If you are monitoring the conduction block on the RPVs and it takes between 2 and 5 applications of your device, how many times are you applying your device on all the other lesions sets, and does that influence what you do on the other side?

Dr Benussi. That is a very good point. Actually, it is the main message from the whole study. Before doing this study with this device, we would do maybe two or three ablations. However, after doing this, I think either you need pacing validation for all the lines, as we said, or you need to do multiple ablations. If you want to do it by eyeballing, you have to base that on the thickness of the tissue you see, how much fat is on the PVs, but never less than three and probably four or five lines around a PV couple. 